\title{
Evaluating multidisciplinary glaucoma care: visual field progression and loss of sight year analysis in the community vs hospital setting
}

\author{
Yusuf Mushtaq $\mathbb{D}^{1}$ - Binita Panchasara ${ }^{2}$ Nikou Nassehzadehtabriz ${ }^{3} \cdot$ Hong Kai Lim $^{3} \cdot$ Maryam Mushtaq $^{3} \cdot$ \\ Jane Kean ${ }^{2} \cdot$ Sarah Farrell ${ }^{2}$ Rupert R. A. Bourne $\mathbb{B}^{2,4} \cdot$ Humma Shahid ${ }^{2} \cdot$ Tasneem Z. Khatib $^{2,5,6}$. \\ Keith R. Martin (iD) $2,5,7,8$
}

Received: 13 October 2020 / Revised: 30 January 2021 / Accepted: 23 February 2021 / Published online: 21 March 2021

(c) The Author(s), under exclusive licence to The Royal College of Ophthalmologists 2021

\begin{abstract}
Background A variety of shared care models have been developed, which aim to stratify glaucoma patients according to risk of disease progression. However, there is limited published data on the rate of glaucoma progression in the hospital vs community setting. Here we aimed to compare rates of glaucomatous visual field progression in the Cambridge Community Optometrist Glaucoma Scheme (COGS) and Addenbrooke's Hospital Glaucoma Clinic (AGC).

Methods A retrospective comparative cohort review was performed. Patients with five or more visual field tests were included. Zeiss Forum software was used to calculate the MD progression rate (dB/year). Loss of sight years (LSY) were also calculated for both COGS and AGC.

Results Overall, 8465 visual field tests from 854 patients were reviewed. In all, 362 eyes from the AGC group and 210 eyes from COGS were included. The MD deterioration rate was significantly lower in the COGS patients compared with the AGC group $(-0.1$ vs $-0.3 \mathrm{~dB}$ /year; $p<0.0001)$. No patients in the COGS group were predicted to become blind within their lifetime by LSY analysis. Fifteen patients were at risk in the AGC group.

Conclusion This service evaluation shows that COGS is an effective scheme to stratify lower risk glaucoma patients, increasing the capacity within hospital eye services. COGS patients have a lower rate of visual field deterioration compared to AGC patients. Effective communication between community and tertiary schemes is essential to facilitate transfer of patients requiring further hospital management reliably and efficiently, with the potential for low-risk patients to be followed safely in the community.
\end{abstract}

\section{Introduction}

The lack of capacity in the United Kingdom's hospital eye services (HES) for glaucoma patients has been highlighted

These authors contributed equally: Tasneem Z. Khatib, Keith R. Martin

Keith R. Martin

keith.martin@unimelb.edu.au

1 Luton and Dunstable University Hospital, Bedfordshire Hospitals NHS Foundation Trust, Luton, UK

2 Eye Department, Cambridge University Hospitals NHS Foundation Trust, Cambridge, UK

3 School of Clinical Medicine, University of Cambridge, Cambridge, UK

4 Vision and Eye Research Institute (VERI), School of Medicine, Anglia Ruskin University, Cambridge, UK in a recent report from the Healthcare Safety Investigation Branch and the award of $£ 3.2$ million in compensation by the National Health Service (NHS) to a 34-year-old glaucoma patient who went blind due to follow-up delays [1]. An estimated 22 people a month suffer severe or permanent loss of sight due to delays in follow-up appointments, and this has also been attributed to insufficient capacity within

5 Department of Clinical Neurosciences, University of Cambridge, Cambridge, UK

6 Medical Sciences Division, University of Oxford, Oxford, UK

7 Ophthalmology, Department of Surgery, University of Melbourne, Melbourne, Australia

8 Centre for Eye Research Australia, Royal Victorian Eye and Ear Hospital, Melbourne, Australia 
HES [2]. The COVID-19 pandemic has further accelerated the need to manage patients away from HES.

Referral rates for glaucoma suspects and ocular hypertensives also increased following the publication of the initial National Institute for Health and Care Excellence (NICE) guidelines for primary open-angle glaucoma (POAG) and ocular hypertension (OHT) [3]. The Royal College of Ophthalmologists (RCOphth) issued national guidance to address the anticipated lack of capacity associated with increased referral rates, recommending that a proportion of glaucoma-related NHS HES attendances were contracted to community-based practitioners [4]. Community-based care particularly applied to patients being monitored for OHT and suspected glaucoma to reduce the HES workload; a case mix representing over $30 \%$ of HES glaucoma-related visits and over 1 million people in England.

A variety of shared care models have been developed in the UK and overseas, which aim to stratify glaucoma patients according to risk of disease progression [5-10]. Often, one goal in such schemes is for hospital-based care to be focused on those at higher risk of disease progression where more aggressive treatment may be required to prevent visual disability [11], with lower risk patients managed outside the hospital according to available local expertise and resources. However, there is limited published data on the rate of glaucoma progression in hospital vs community setting for many of these schemes. In the current study, our aim was to conduct a service evaluation to compare rates of glaucomatous visual field progression and loss of sight years (LSY) in the Cambridge Community Optometrist Glaucoma Scheme (COGS) and Addenbrooke's Hospital Glaucoma Clinic (AGC). The AGC is a tertiary referral centre, but as there is no other hospital serving the local population, the glaucoma clinic for adult patients also functions in a very similar way to other district general hospitals in addition to providing specialist services. We therefore feel that our study is applicable to both secondary and tertiary level services.

\section{The Cambridge Community Optometrist Glaucoma Scheme (COGS)}

COGS was established in 2010 and initially used for referral refinement [10]. All new referrals to AGC were triaged by a glaucoma specialist nurse into high-risk and low-risk categories. Those deemed low risk (intraocular pressure (IOP) below $30 \mathrm{mmHg}$ or a field defect suspicious of glaucoma or an optic disc suspicious of glaucoma) were assessed in COGS. In 2012, the scheme expanded to monitor ocular hypertensive and glaucoma suspect patients (treated and untreated) and in 2015 it was expanded further to allow the monitoring of patients with early POAG, considered low risk for vision loss. In 2019, the scheme widened the inclusion criteria to include more complex- and moderaterisk glaucoma cases. The current risk stratification guideline used to determine if a patient is suitable for COGS followup is outlined in Table 1. This guideline is based on an agreed empiric assessment of risk, in conjunction with local stakeholders and clinicians. Changes to the NICE guidelines for the diagnosis and management in glaucoma in 2017, including an increased emphasis on optic disc imaging which was not available uniformly in our community clinics, led to a modification of the scheme such that all newly referred patients are now first assessed in AGC with baseline imaging and then referred to the COGS scheme if appropriate.

Patients are examined according to a local protocol using equipment standardised to AGC. Due to a lack of uniform capacity for ocular coherence tomography (OCT) in community optometry practices participating in COGS during the period reported, OCT was not mandated in the COGS clinical assessment, but was performed in the AGC. Participating optometrists complete training in glaucoma under the supervision of a consultant ophthalmologist. They are also required to complete the College of Optometrists Professional Certificate in Glaucoma [12].

Community optometrists have remote access to hospital electronic records and enter all their results in real time. Findings are summarised using a standardised template. All results are reviewed by a consultant ophthalmologist or hospital optometrist within 4 weeks of the community glaucoma assessment. An outcome letter is generated by the reviewing clinician and copies sent to the GP and patient within 6 weeks of the community glaucoma assessment.

\section{Methods}

A retrospective comparative cohort review was performed of patients attending the AGC clinic or COGS clinic. All patients had SITA-standard automated perimetry performed with the Humphrey Field Analyzer (Carl Zeiss Meditec, Dublin, California) using the 24-2 full-threshold programme with a Goldmann size III stimulus and appropriate refractive correction.

Patients that had either had one or two eyes with five or more SITA-standard 24-2 visual field tests were included in the analysis. All patients from the COGS service since its inception in 2010-2019 and a similar cohort from the AGC group were analysed. Data for each eye were processed separately where both eyes were included. The Zeiss Forum software was used to extract the data and calculate the MD progression rate (dB/year) using Guided Progression Analysis. Graphpad Prism software was used for statistical 
Table 1 The Cambridge Community Optometry Glaucoma Scheme (COGS).

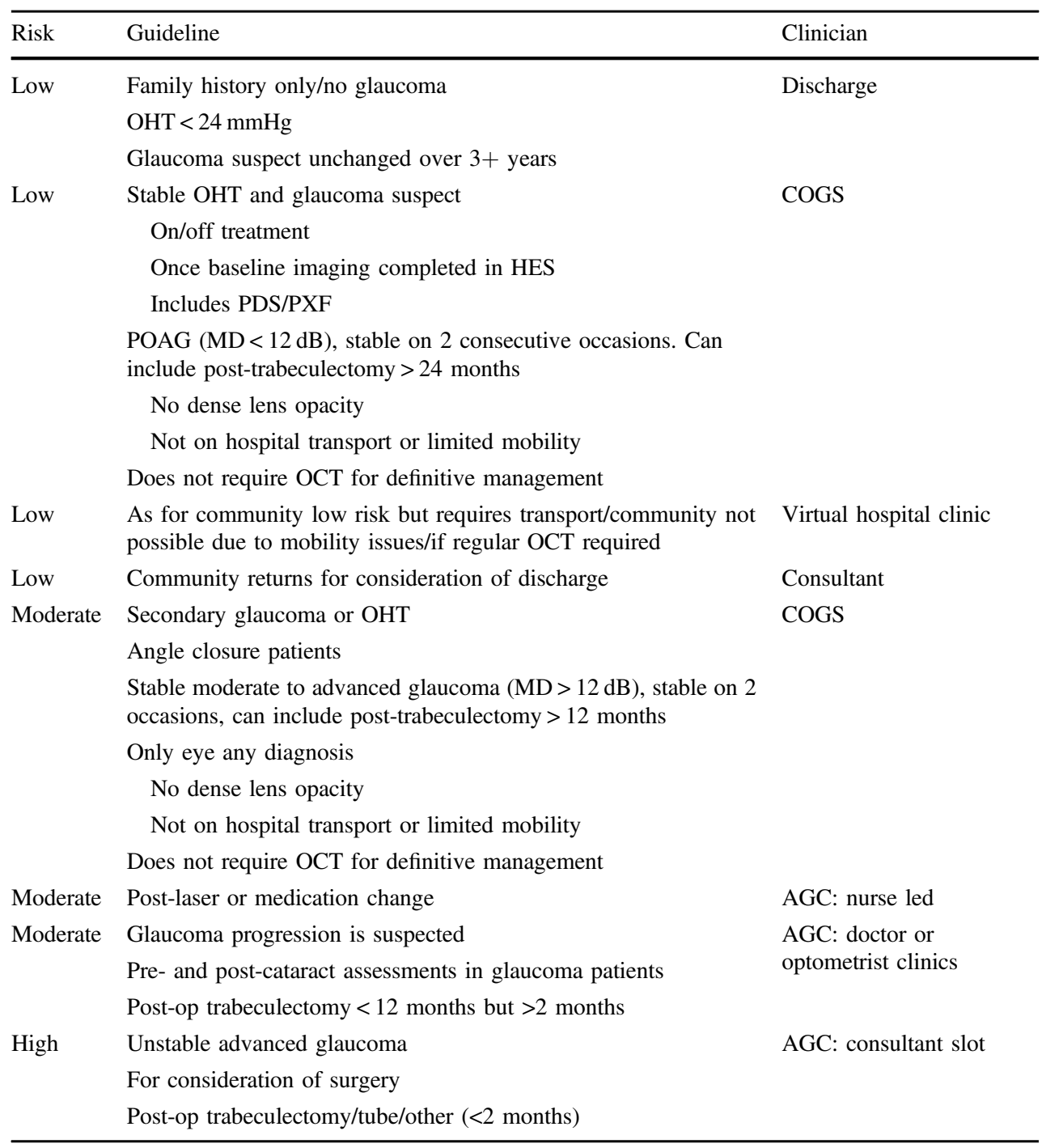

analysis. The D'Agostino-Pearson test was used to evaluate whether the distribution of continuous variables was normal. The descriptive statistics for non-normally distributed variables (number of visual field tests, follow-up, mean deviation rate of progression, LSY) are presented as median (25th-75th percentiles) unless otherwise specified. The Mann-Whitney test was used to compare non-normally distributed independent variables between groups. LSY analysis was performed by estimating the number of years each patient would develop bilateral VF loss worse than $\mathrm{MD}$ of $-22 \mathrm{~dB}$ in their predicted remaining lifetime as previously described [13].

\section{Results}

In all, 8465 visual field tests from a total of 854 patients were reviewed. Overall, 362 eyes from the AGC group and 210 eyes from the COGS group met the eligibility criteria of five or more SITA-standard 24-2 visual field tests. All diagnoses and visual field tests performed were included in the analysis (Table 2).

The median MD rates highlighted worsening vision over time in both groups (Table 3). However, the rate of deterioration was significantly lower in the COGS patients compared with the AGC group ( -0.1 vs $-0.3 \mathrm{~dB} /$ year; $p<0.0001)$.

COGS followed up the majority of patients progressing between 0 and $1 \mathrm{~dB}$ per year. In all, $14.3 \%$ of patients in the AGC were progressing at a rate $>1 \mathrm{~dB} /$ year compared to $6.2 \%$ in the COGS group. Of the 13 patients in the COGS service that were progressing at $>1 \mathrm{~dB}$ /year, $11(87 \%)$ had additional non-glaucoma diagnoses that would also affect the visual field. There were no patients in the COGS group deteriorating at rates $>3 \mathrm{~dB} /$ year.

In general, ocular comorbidities that may have affected the visual field were relatively similar across both groups including age-related macular degeneration (4.4\% in AGC 
Table 2 Summary statistics.

\begin{tabular}{|c|c|c|}
\hline & Tertiary (AGC) & Community (COGS) \\
\hline Number of eyes & 362 & 210 \\
\hline Number of patients & 188 & 119 \\
\hline Age, years $($ mean \pm SEM $)$ & $74.8( \pm 0.80)$ & $69.9( \pm 0.98)$ \\
\hline \multicolumn{3}{|l|}{ Gender, $n(\%)$} \\
\hline Male & $100(53.2)$ & $69(57.9)$ \\
\hline Female & $88(46.8)$ & $50(42.1)$ \\
\hline Median number of VF tests & $7(6-10)$ & $6(5-8)$ \\
\hline Median follow-up, years & $8(4-11)$ & $5(4-8)$ \\
\hline Baseline severity MD & $\begin{array}{l}-2.43 \\
(-5.8 \text { to }-0.8)\end{array}$ & $\begin{array}{l}-2.08 \\
(-4.9 \text { to }-0.4)\end{array}$ \\
\hline \multicolumn{3}{|l|}{ Diagnosis, $n(\%)$} \\
\hline $\begin{array}{l}\text { Primary open-angle } \\
\text { glaucoma }\end{array}$ & $194(53.8)$ & $140(66.2)$ \\
\hline Ocular hypertension & $39(10.9)$ & $24(11.3)$ \\
\hline $\begin{array}{l}\text { Primary angle closure } \\
\text { glaucoma }\end{array}$ & $24(6.7)$ & 0 \\
\hline $\begin{array}{l}\text { Normal tension } \\
\text { glaucoma }\end{array}$ & $44(12.0)$ & $34(16.4)$ \\
\hline Glaucoma suspect & $19(5.3)$ & $4(1.9)$ \\
\hline $\begin{array}{l}\text { Secondary open-angle } \\
\text { glaucoma }\end{array}$ & $26(7.2)$ & $4(1.9)$ \\
\hline Neovascular glaucoma & $3(0.8)$ & $2(0.9)$ \\
\hline Uveitic glaucoma & $11(3.1)$ & $3(1.4)$ \\
\hline Juvenile onset glaucoma & $1(0.3)$ & 0 \\
\hline
\end{tabular}

vs $2.9 \%$ in COGS) and diabetic retinopathy (1.1\% in AGC vs $3.8 \%$ in COGS). Overall, $32.9 \%$ of AGC patients had undergone cataract surgery vs $21.4 \%$ in COGS.

We also evaluated the visual field loss at the end of the study (Fig. 1A-C). We defined early glaucoma as a visual field defect corresponding to a MD of $-6 \mathrm{~dB}$ or better, moderate glaucoma as an MD between -6 and $-12 \mathrm{~dB}$, severe glaucoma as an MD between -12 and $-20 \mathrm{~dB}$ and end-stage glaucoma as an MD of $-20 \mathrm{~dB}$ or worse. COGS had a greater proportion of patients with early glaucoma (56.4\% in AGC vs $72.9 \%$ in COGS), while AGC had a greater proportion of patients with moderate glaucoma (26.5\% in AGC vs $13.8 \%$ in COGS). Interestingly, there was a similar percentage of patients with severe glaucoma in both groups (11\% in AGC vs $10 \%$ in COGS). The proportion of patients with end-stage glaucoma in AGC was approximately double that seen in COGS (6.1 vs 3.3\%). The proportion of patients in each group who did not have glaucomatous visual field loss at entry was $16 \%$ in AGC and $19.5 \%$ in COGS. At the end point of the study, these proportions were $10.2 \%$ in AGC and $18.1 \%$ in COGS. There was a significant correlation between the visual field loss observed at the end of the study and the rate of MD progression across both groups although the correlation
Table 3 Distribution of MD progression rates.

\begin{tabular}{lll}
\hline & Tertiary (AGC) & Community (COGS) \\
\hline MD progression $\mathrm{dB} / \mathrm{yr}$ & $-0.3(-0.7$ to 0.0$)$ & $-0.1(-0.4$ to 0.1$)$ \\
Range & -6.3 to 1.1 & -2.1 to 1.3 \\
$-0.5<x \leq-0.1$ & $134 / 362(37.0 \%)$ & $141 / 210(67.1 \%)$ \\
$-1<x \leq-0.5$ & $78 / 362(21.5 \%)$ & $27 / 210(12.9 \%)$ \\
$-2<x \leq-1$ & $40 / 362(11.0 \%)$ & $12 / 210(5.7 \%)$ \\
$-3<x \leq-2$ & $7 / 362(1.9 \%)$ & $1 / 210(0.5 \%)$ \\
$-4<x \leq-3$ & $1 / 362(0.3 \%)$ & $0 / 210(0 \%)$ \\
$x \leq-4$ & $4 / 362(1.1 \%)$ & $0 / 210(0 \%)$ \\
\hline
\end{tabular}

was weaker for COGS patients (Spearman's correlation coefficient $r=0.60$ for AGC vs 0.33 for COGS; $p<0.0001$ in both groups). No significant correlation was seen between the initial MD and rate of MD progression (Spearman's correlation coefficient $r=-0.07$ for AGC; $p=0.16$ vs $r=0.03$ for COGS; $p=0.66$ ) or the baseline severity between both groups $(p=0.15)$. Figure 1D shows the relationship between initial MD and rate of MD progression across both groups.

The LSY analysis was performed in patients from whom data from both eyes was available (Fig. 2). This showed a median of 0 years $(0-0)$ in both groups, suggesting that the majority of patients in either group were unlikely to become blind during the course of their lifetime. There were no rapid progressors in the COGS group and no patients in the COGS group were at risk of becoming blind within their lifetime. Fifteen patients were at risk in the AGC group. Weak correlation was seen between the presenting MD and LSY (Spearman's correlation coefficient $r=-0.13 ; p=0.04$ ).

\section{Discussion}

COGS was established to help streamline the management of glaucoma in Cambridge, aiming to concentrate higher risk glaucoma patients in AGC and lower risk patients in community clinics. In the current study, there was a significantly greater rate of MD progression in the AGC compared to COGS. No patients were identified as at risk of becoming blind in their lifetime in the COGS group and all rapid progressors (progressing at $>3 \mathrm{~dB} /$ year) were followed up in AGC. COGS also followed up a greater proportion of slow progressors (between 0 and $1 \mathrm{~dB} /$ year) and 11 of 13 patients in the COGS group progressing at $>1 \mathrm{~dB} /$ year had other pathologies such as cataract, or coexisting neurological or retinal pathology. One patient from the COGS group progressing at $>1 \mathrm{~dB}$ /year also requested to remain under the COGS service rather transfer back to AGC.

Ongoing capacity pressures within AGC have led to an increase in the proportion of patients with more advanced 
A

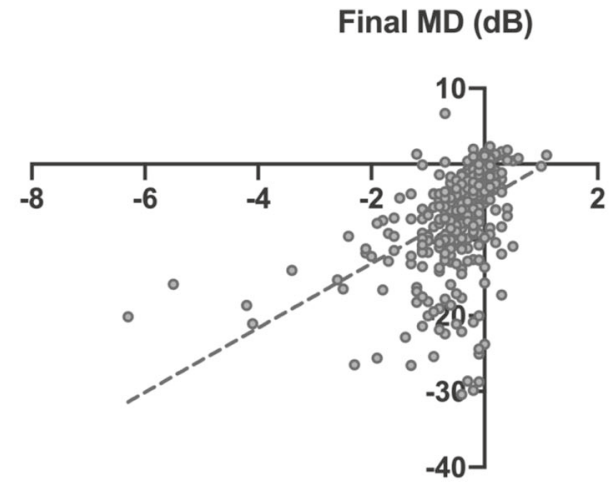

MD progression rate ( $\mathrm{dB} /$ year)

C
B

\section{Final MD (dB)}

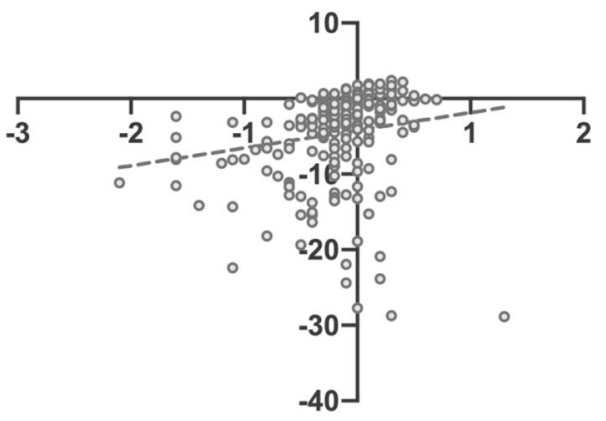

MD progression rate (dB/year)

\begin{tabular}{|ccc|}
\hline Final MD (dB) & Tertiary (AGC) & Community (COGS) \\
\hline $\mathbf{x} \geq \mathbf{- 6}$ & $204 / 362(56.4 \%)$ & $153 / 210(72.9 \%)$ \\
\hline $\mathbf{- 1 2} \leq \mathbf{x}<-\mathbf{6}$ & $96 / 362(26.5 \%)$ & $29 / 210(13.8 \%)$ \\
\hline $\mathbf{- 2 0} \leq \mathbf{x}<-\mathbf{1 2}$ & $40 / 362(11.0 \%)$ & $21 / 210(10 \%)$ \\
\hline $\mathbf{x}<-\mathbf{2 0}$ & $22 / 362(6.1 \%)$ & $7 / 210(3.3 \%)$ \\
\hline
\end{tabular}

D

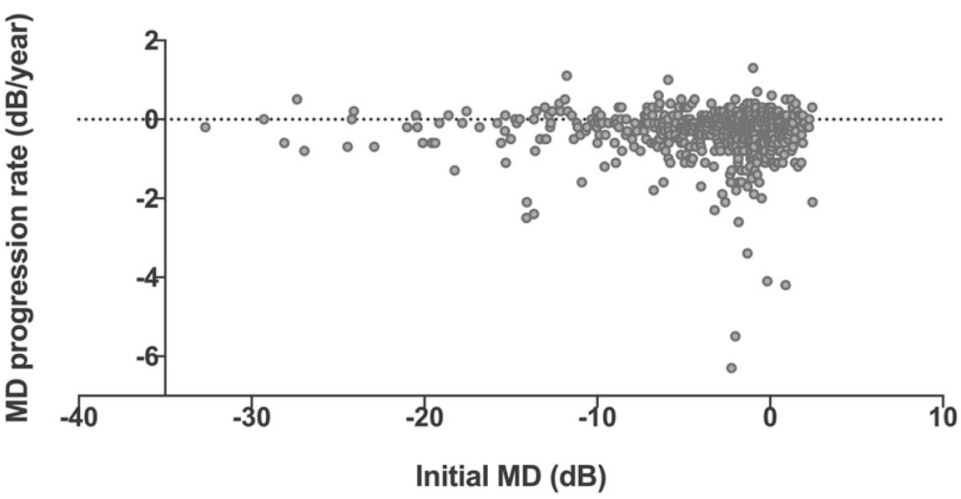

Fig. 1 Visual field loss and MD progression rate. A Relationship between visual field loss at end of study and rate of MD progression in AGC patients. Spearman's correlation coefficient $r=0.60 ; p<0.0001$.

B Relationship between visual field loss at end of study and rate of

glaucoma that are followed within the community scheme over time. This highlights the need for effective communication channels to identify patients requiring further hospital management reliably and efficiently. A recent internal audit conducted at the end of the period of analysis demonstrated that $41 \%$ of all patients $(45 \%$ of moderate-risk patients) monitored in COGS were deemed to be unstable at virtual review and required an AGC appointment. Upon
MD progression in COGS patients. Spearman's correlation coefficient $r=0.33 ; p<0.0001$. C Distribution of final MD. D Relationship between initial MD and rate of MD progression.

review in hospital, the majority of those patients (72\%) were correctly identified as unstable and required a change of treatment or closer AGC monitoring.

The widening scope of patients managed in COGS could be considered a strength of the scheme. Rather than exclude this higher risk cohort from community clinics, strategies continue to be developed to manage these patients in the community. The planned transition to virtual amendment of 

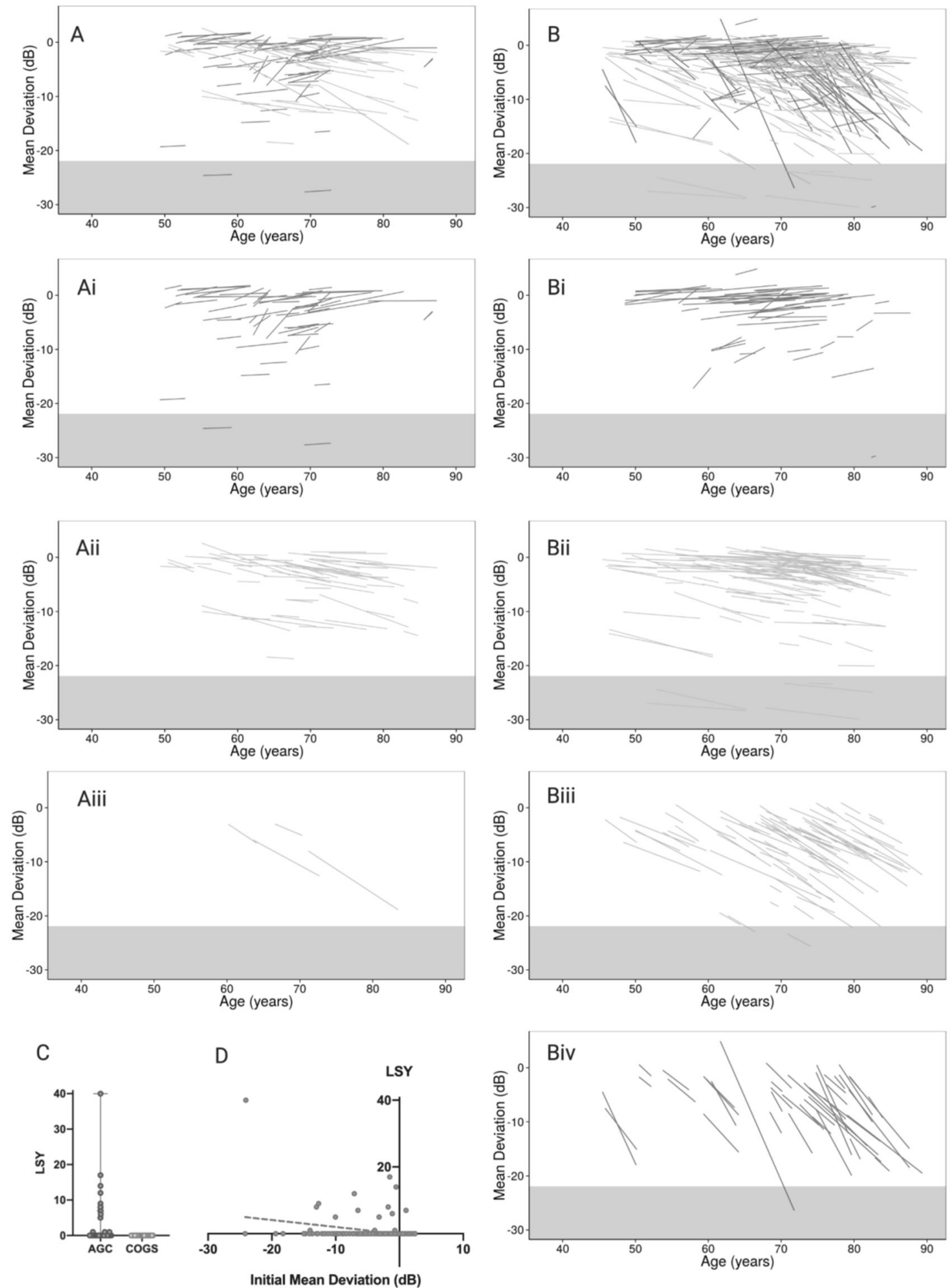

Fig. 2 Loss of sight year (LSY) analysis. A Hedgehog plot for all COGS patients: Ai stable COGS patients, Aii slow COGS progressors, Aiii moderate COGS progressors. B Hedgehog plot for all AGC patients: Bi stable AGC patients, Bii slow AGC progressors, Biii

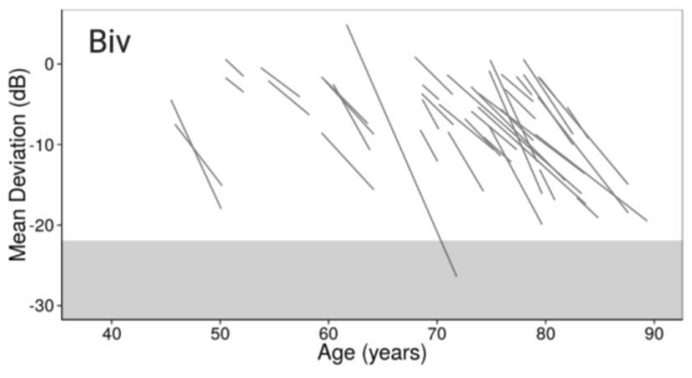

moderate AGC progressors Biv rapid AGC progressors. C LSY in AGC and COGS. D Correlation between initial MD and LSY (Spearman-s correlation coefficient $r=-0.13 ; p=0.04$ ).

treatment changes by the clinical reviewer with subsequent COGS follow-up has the potential to reduce the AGC referral rate. Our risk stratification is based on empiric

assessment in conjunction with local stakeholders and clinicians. Alternative stratification methods also include recent tools published by the RCOphth and UK and Eire 
Glaucoma Society [14] and the UK Ophthalmology Alliance [15] although it is likely that these guidelines will be tailored according to regional needs and available resources.

LSY analysis was used to help assess the safety of following patients predicted to be at low risk in primary care rather than hospital glaucoma clinics. However, it is important to remember that patients can also suffer a reduced quality of life with an increased risk of falls and other comorbidity due to visual loss before blindness and therefore LSY should not be used as a tool in isolation and the results should be interpreted with caution.

Streamlining and stratifying pathways to facilitate the management of an increasing glaucoma population are essential with community services continuing to grow in response to service need. However, a proportion of patients who have access to regular, high-quality care also continue to deteriorate. It is therefore not surprising that patients followed in COGS still have a detectable rate of glaucoma progression.

The overall median rate of deterioration of $-0.3 \mathrm{~dB} / \mathrm{year}$ in AGC and $-0.1 \mathrm{~dB} /$ year in COGS is comparable to previous reports of glaucoma progression in UK secondary care [16], as is the proportion of patients progressing at a rate $>1 \mathrm{~dB} /$ year $(14.2 \%$ in the AGC group). Our rates are lower than those reported from a large tertiary glaucoma service in Sweden (median - $0.62 \mathrm{~dB} /$ year) [17], although that population has a higher proportion of patients with pseudoexfoliation glaucoma who are known to have a higher risk of progression [18, 19]. Another factor contributing to this difference between centres may be that our data were extracted from either eye, whereas the Lund authors used data from the eye with the greater visual field defect.

Large multi-centre trials such as the Early Manifest Glaucoma Trial [19] and the United Kingdom Glaucoma Treatment Study [20] also found that a significant proportion of patients in the treatment arms progressed. Moreover, $30-40 \%$ or more of glaucoma patients in many populations show normal tension in IOP measurements [21]. In Japan, normal tension glaucoma (NTG) accounts for the majority of patients diagnosed with open-angle glaucoma [22]. Although aggressively lowering the IOP by $30 \%$ has been shown to reduce the risk of progression for patients with NTG from 35 to $12 \%$ over 5 years, this still means a large number of people will continue to lose vision despite best available current treatment.

The reasons why retinal ganglion cell loss and corresponding visual field deterioration continue despite best treatment are currently unknown, but contributing factors may include insufficient IOP lowering with conventional treatment, poor compliance with intended therapy or progression despite optimised IOP lowering treatment. A variety of approaches are being undertaken to tackle these problems, from the refinement of medical, laser and surgical approaches to lower the IOP [23-29], understanding genetic predisposition and the development of personalised medicine $[30,31]$, to new sustained release drug-delivery devices [32] to minimise the effect of poor compliance and the development of neuroprotective strategies as an alternative or adjunct to IOP lowering therapies [33].

Another consideration is the usefulness of OCT scanning in the assessment of glaucoma in the community setting. Abnormalities on OCT scanning of the optic nerve head in subjects over 50 years have been reported to have a false positive glaucoma detection rate of $30 \%$ [34]. In another study, focal abnormalities of the retinal nerve fibre layer on spectral domain OCT scanning had a false positive rate of $35 \%$ [35]. Furthermore, each OCT technology has different analysis software which is not interchangeable between machines. We therefore did not mandate OCT in the community setting at the time the current scheme was established, although this is currently being reassessed.

For the future, artificial intelligence techniques are playing a key role and their integration into clinical practice has the potential to significantly improve our early detection of at-risk patients and enhance our understanding of the underlying pathophysiology of glaucomatous visual loss [36-38]. More accurate endpoints to monitor disease progression and the effects of intervention will also be important [33, 39]. A number of functional outcome measures are being evaluated in the assessment of glaucoma patients in addition to visual field testing [40-44], from contrast sensitivity [45, 46], electrophysiology [47], task completion or simulation [48, 49] to patient reported experience of visual loss [50-52].

COGS appears an effective scheme to help manage lower risk glaucoma patients in the UK environment where accredited optometric support can be developed outside of the hospital setting, which increases the capacity within HES to manage higher risk patients. COGS patients have a lower rate of visual field deterioration compared to patients seen in AGC. Effective communication between the community and tertiary schemes is essential to facilitate transfer of patients requiring further hospital management safely and efficiently.

\section{Summary}

\section{What was known before}

- A variety of shared care models have been developed which aim to stratify glaucoma patients according to risk of disease progression.

- However, there is limited published data on the rate of glaucoma progression in the hospital vs community setting. 


\section{What this study adds}

- The Cambridge Community Optometry Glaucoma Scheme is an effective scheme to stratify lower risk glaucoma patients, increasing the capacity within hospital eye services.

- Community patients have a lower rate of visual field deterioration compared to hospital patients.

- Effective communication between community and tertiary schemes is essential to facilitate transfer of patients requiring further hospital management reliably and efficiently, with the potential for low-risk patients to be followed safely in the community.

Acknowledgements We would like to thank Dr. Susan Bryan and Professor David Crabb, City University for their guidance when performing the loss of sight year analysis and the Cambridge University Hospitals NHS Foundation Trust information analyst team for their technical assistance. We would also like to thank the community optometrists involved in the COGS scheme: Anjana Taank (Taanks Optometrists), Maria Tipaldi (Anglia Ruskin University), John Kidd (Anglia Ruskin University), Kam Dhillon (Specsavers).

\section{Compliance with ethical standards}

Conflict of interest The authors declare no competing interests.

Publisher's note Springer Nature remains neutral with regard to jurisdictional claims in published maps and institutional affiliations.

\section{References}

1. Healthcare Safety Investigation Branch. Lack of timely monitoring of patients with glaucoma. Healthcare Safety Investigation 2019/001. United Kingdom: Healthcare Safety Investigation Branch; 2020.

2. Foot B, MacEwen C. Surveillance of sight loss due to delay in ophthalmic treatment or review: frequency, cause and outcome. Eye. 2017;31:771-5. https://doi.org/10.1038/eye.2017.1.

3. Chalk D, Smith M. Guidelines on glaucoma and the demand for services. Br J Health Manag. 2013;19:476-81. https://doi.org/10. 12968/bjhc.2013.19.10.476.

4. Royal College of Ophthalmologists. Commissioning guide: glaucoma. 2016. Available from: https://www.rcophth.ac.uk/wpcontent/uploads/2016/06/Glaucoma-Commissioning-Guide-LongJune-2016-Final.pdf.

5. Vernon SA, Adair A. Shared care in glaucoma: a national study of secondary care lead schemes in England. Eye. 2010;24:265-9.

6. Gray SF, Spry PGD, Brookes ST, Peters TJ, Spencer IC, Baker IA, et al. The Bristol shared care glaucoma study: outcome at follow up at 2 years. Br J Ophthalmol. 2000;84:456-63. http://bjo. bmj.com/content/84/5/456.abstract.

7. Gray SF, Spencer IC, Spry PG, Brookes ST, Baker IA, Peters TJ, et al. The Bristol shared care glaucoma study-validity of measurements and patient satisfaction. J Public Health. 1997;19:431-6. https://doi.org/10.1093/oxfordjournals.pubmed.a024673.

8. Mandalos A, Bourne R, French K, Newsom W, Chang L. Shared care of patients with ocular hypertension in the Community and
Hospital Allied Network Glaucoma Evaluation Scheme (CHANGES). Eye. 2012;26:564-7. https://doi.org/10.1038/eye.2011.350.

9. Kotecha A, Brookes J, Foster PJ. A technician-delivered 'virtual clinic' for triaging low-risk glaucoma referrals. Eye. 2017;31: 899-905. https://doi.org/10.1038/eye.2017.9.

10. Keenan J, Shahid H, Bourne RR, White AJ, Martin KR. Cambridge community optometry glaucoma scheme. Clin Exp Ophthalmol. 2015;43:221-7. https://doi.org/10.1111/ceo.12398.

11. Caprioli J. The importance of rates in glaucoma. Am J Ophthalmol. 2008;145:191-2. https://doi.org/10.1016/j.ajo.2007.12.003.

12. The College of Optometrists. Learning outcomes for the professional certificate in glaucoma. 2018. Available from: https://www. college-optometrists.org/uploads/assets/0e49d21f-7691-4e1c-a d46f8b4ac29268e/Learning-outcomes-Professional-Certificate-inGlaucoma.pdf.

13. Bryan SR, Crabb DP. A new graphical tool for assessing visual field progression in clinical populations. Transl Vis Sci Technol. 2018;7:22. https://pubmed.ncbi.nlm.nih.gov/29497584.

14. RCOphth and UKEGS. Joint RCOphth and UKEGS glaucoma risk stratification tool. 2020. Available from: https://www.rcophth. ac.uk/wp-content/uploads/2020/08/Glaucoma-Risk-StratificationTool-1.pdf.

15. UKOA. How to stratify the risk or complexity for glaucoma to direct patients to appropriate clinics. 2020. Available from: https:// uk-oa.co.uk/wp-content/uploads/2020/02/Glaucoma-Risk-andVirtuals_PDF.pdf.

16. Kirwan JF, Hustler A, Bobat H, Toms L. Portsmouth visual field database: an audit of glaucoma progression. 2014;28:974-9. https://doi.org/10.1038/eye.2013.294.

17. Heijl A, Buchholz P, Norrgren G, Bengtsson B. Rates of visual field progression in clinical glaucoma care. Acta Ophthalmol. 2013;91:406-12. https://doi.org/10.1111/j.1755-3768.2012.02492.x.

18. Moon Y, Sung KR, Kim JM, Shim SH, Yoo C, Park JH. Risk factors associated with glaucomatous progression in pseudoexfoliation patients. J Glaucoma. 2017;26:1107-13.

19. Leske MC, Heijl A, Hussein M, Bengtsson B, Hyman L, Komaroff E, et al. Factors for glaucoma progression and the effect of treatment: the early manifest glaucoma trial. Arch Ophthalmol. 2003;121:48-56. https://doi.org/10.1001/archopht.121.1.48.

20. Garway-Heath DF, Crabb DP, Bunce C, Lascaratos G, Amalfitano $\mathrm{F}$, Anand $\mathrm{N}$, et al. Latanoprost for open-angle glaucoma (UKGTS): a randomised, multicentre, placebo-controlled trial. Lancet. 2015;385:1295-304.

21. Kamal D, Hitchings R. Normal tension glaucoma-a practical approach. Br J Ophthalmol. 1998;82:835-40. https://www.ncbi. nlm.nih.gov/pubmed/9924383.

22. Cho H, Kee C. Population-based glaucoma prevalence studies in Asians. Surv Ophthalmol. 2014;59:434-47. https://doi.org/10. 1016/j.survophthal.2013.09.003.

23. Tanna AP, Johnson M. Rho kinase inhibitors as a novel treatment for glaucoma and ocular hypertension. Ophthalmology. 2018;125: 1741-56. https://doi.org/10.1016/j.ophtha.2018.04.040.

24. Walters TR, Ahmed IIK, Lewis RA, Usner DW, Lopez J, Kopczynski CC, et al. Once-daily netarsudil/latanoprost fixeddose combination for elevated intraocular pressure, the randomized phase 3 MERCURY-2 study. Ophthalmol Glaucoma. 2019. https://linkinghub.elsevier.com/retrieve/pii/S2589419619300997.

25. Le JT, Bicket AK, Wang L, Li T. Ab interno trabecular bypass surgery with iStent for open-angle glaucoma. Cochrane Database Syst Rev. 2019;3:CD012743.

26. Sieck EG, Epstein RS, Kennedy JB, SooHoo JR, Pantcheva MB, Patnaik JL, et al. Outcomes of Kahook Dual Blade goniotomy with and without phacoemulsification cataract extraction. Ophthalmol Glaucoma. 2018;1:75-81.

27. Denis P, Hirneiß C, Reddy KP, Kamarthy A, Calvo E, Hussain Z, et al. A first-in-human study of the efficacy and safety of MINIject 
in patients with medically uncontrolled open-angle glaucoma (STAR-I). Ophthalmol Glaucoma. 2019;2:290-7.

28. Samuelson TW, Chang DF, Marquis R, Flowers B, Lim KS, Ahmed IIK, et al. A schlemm canal microstent for intraocular pressure reduction in primary open-angle glaucoma and cataract: the HORIZON study. Ophthalmology. 2019;126:29-37.

29. Sarrafpour S, Saleh D, Ayoub S, Radcliffe NM. Micropulse transscleral cyclophotocoagulation: a look at long-term effectiveness and outcomes. Ophthalmol Glaucoma. 2019;2:167-71. http://www. sciencedirect.com/science/article/pii/S2589419618302163.

30. Craig JE, Han X, Qassim A, Hassall M, Cooke Bailey JN, Kinzy TG, et al. Multitrait analysis of glaucoma identifies new risk loci and enables polygenic prediction of disease susceptibility and progression. Nat Genet. 2020;52:160-6.

31. Qassim A, Souzeau E, Siggs OM, Hassall MM, Han X, Griffiths $\mathrm{HL}$, et al. An intraocular pressure polygenic risk score stratifies multiple primary open-angle glaucoma parameters including treatment intensity. Ophthalmology. 2020;127:901-7.

32. Lewis RA, Christie WC, Day DG, Craven ER, Walters T, Bejanian $\mathrm{M}$, et al. Bimatoprost sustained-release implants for glaucoma therapy: 6-month results from a phase I/II clinical trial. Am J Ophthalmol. 2017;175:137-47. http://www.sciencedirect.com/ science/article/pii/S0002939416305955.

33. Khatib TZ, Martin KR. Neuroprotection in glaucoma: towards clinical trials and precision medicine. Curr Eye Res. 2020;45:327-38. https://doi.org/10.1080/02713683.2019.1663385.

34. Healey PR, Lee AJ, Aung T, Wong TY, Mitchell P. Diagnostic accuracy of the heidelberg retina tomograph for glaucoma: a population-based assessment. Ophthalmology. 2010;117:1667-73. http://www.sciencedirect.com/science/article/pii/S0161642010007165.

35. Bussel II, Wollstein G, Schuman JS. OCT for glaucoma diagnosis, screening and detection of glaucoma progression. Br J Ophthalmol. 2014;98:ii15-9. http://bjo.bmj.com/content/98/Suppl_2/ii15.abstract.

36. Yousefi S, Elze T, Pasquale LR, Saeedi O, Wang M, Shen LQ, et al. Monitoring glaucomatous functional loss using an artificial intelligence-enabled dashboard. Ophthalmology. 2020. http://www. sciencedirect.com/science/article/pii/S0161642020302384.

37. Yousefi S, Kiwaki T, Zheng Y, Sugiura H, Asaoka R, Murata $\mathrm{H}$, et al. Detection of longitudinal visual field progression in glaucoma using machine learning. Am J Ophthalmol. 2018;193:71-9.

38. Thakur A, Goldbaum M, Yousefi S. Predicting glaucoma before onset using deep learning. Ophthalmol Glaucoma. 2020;3:262-8. http://www.sciencedirect.com/science/article/pii/ S2589419620301071.

39. Moraes CGDE, Liebmann JM, Levin LA. Detection and measurement of clinically meaningful visual field progression in clinical trials for glaucoma. Prog Retin Eye Res. 2017;56:107-47.

40. Johnson CA. Short-wavelength automated perimetry in low-, medium-, and high-risk ocular hypertensive eyes. Arch
Ophthalmol. 1995;113:70. http://archopht.jamanetwork.com/a rticle.aspx?doi=10.1001/archopht.1995.01100010072023.

41. Beck RW, Bergstrom TJ, Lighter PR. A clinical comparison of visual field testing with a new automated perimeter, the Humphrey Field Analyzer, and the goldmann perimeter. Ophthalmology. 1985;92:77-82. https://www.sciencedirect.com/science/article/pii/ S0161642085340654.

42. Johnson CA. Blue-on-yellow perimetry can predict the development of glaucomatous visual field loss. Arch Ophthalmol. 1993;111:645. http://archopht.jamanetwork.com/a rticle.aspx?doi=10.1001/archopht.1993.01090050079034.

43. Bayer AU, Erb C. Short wavelength automated perimetry, frequency doubling technology perimetry, and pattern electroretinography for prediction of progressive glaucomatous standard visual field defects. Ophthalmology. 2002;109:1009-17. https://www.sciencedirect.com/ science/article/pii/S0161642002010151.

44. Landers JA, Goldberg I, Graham SL. Detection of early visual field loss in glaucoma using frequency-doubling perimetry and shortwavelength automated perimetry. Arch Ophthalmol. 2003;121: 1705-10. https://doi.org/10.1001/archopht.121.12.1705.

45. Richman J, Lorenzana LL, Lankaranian D, Dugar J, Mayer J, Wizov $\mathrm{SS}$, et al. Importance of visual acuity and contrast sensitivity in patients with glaucoma. Arch Ophthalmol. 2010;128:1576-82. https://doi.org/10.1001/archophthalmol.2010.275.

46. Mckendrick AM, Sampson GP, Walland MJ, Badcock DR. Contrast sensitivity changes due to glaucoma and normal aging: low-spatial-frequency losses in both magnocellular and parvocellular pathways. Invest Ophthalmol Vis Sci. 2007;48:2115-22. https://doi.org/10.1167/iovs.06-1208.

47. Wilsey LJ, Fortune B. Electroretinography in glaucoma. Curr Opin Ophthalmol. 2016;27:118-24.

48. Skalicky SE, Mcalinden C, Khatib T, Anthony LM, Sim SY, Martin KR, et al. Activity limitation in glaucoma: objective assessment by the Cambridge Glaucoma Visual Function Test. Invest Ophthalmol Vis Sci. 2016;57:6158-66. https://doi.org/10. 1167/iovs.16-19458.

49. Goh RLZ, Kong YXG, McAlinden C, Liu J, Crowston JG, Skalicky SE. Objective Assessment of Activity Limitation in Glaucoma with Smartphone Virtual Reality Goggles: A Pilot Study. Transl Vis Sci Technol. 2018;7:10. https://doi.org/10.1167/tvst.7.1.10.

50. Crabb DP, Smith ND, Glen FC, Burton R, Garway-Heath DF. How does glaucoma look? Patient perception of visual field loss. Ophthalmology. 2013;120:1120-6. https://doi.org/10.1016/j. ophtha.2012.11.043.

51. Skalicky SE, Lamoureux EL, Crabb DP, Ramulu PY. Patientreported outcomes, functional assessment, and utility values in glaucoma. J Glaucoma. 2019;28:89-96.

52. Somner JEA, Sii F, Bourne RR, Cross V, Burr JM, Shah P. Moving from PROMs to POEMs for glaucoma care: a qualitative scoping exercise. Invest Ophthalmol Vis Sci. 2012;53:5940-7. 